\title{
BIFURCATION GAPS IN ASYMMETRIC AND HIGH-DIMENSIONAL HYPERCYCLES*
}

\author{
JÚLIA PUIG; GERARD FARRÉ \\ ERNEST FONTICH, and JOSEP SARDANYÉS"
}

April 24, 2018

\begin{abstract}
Hypercycles are catalytic systems with cyclic architecture. These systems have been suggested to play a key role in the maintenance and increase of information in prebiotic replicators. It is known that for a large enough number of hypercycle species $(n>4)$ the coexistence of all hypercycle members is governed by a stable periodic orbit. Previous research has characterized saddle-node ( $\mathrm{s}-\mathrm{n}$ ) bifurcations involving abrupt transitions from stable hypercycles to extinction of all hypercycle members, or, alternatively, involving the outcompetition of the hypercycle by so-called mutant sequences or parasites. Recently, the presence of a bifurcation gap between a s-n bifurcation of periodic orbits and a s-n of fixed points has been described for symmetric five-member hypercycles. This gap was found between the value of the replication quality factor $Q$ from which the periodic orbit vanishes $\left(Q_{P O}\right)$ and the value where two unstable (nonzero) equilibrium points collided $\left(Q_{S S}\right)$. Here, we explore the persistence of this gap considering asymmetries in replication rates in five-member hypercycles as well as considering symmetric, larger hypercycles. Our results indicate that both the asymmetry in Malthusian replication constants and the increase in hypercycle members enlarge the size of this
\end{abstract}

*Version previous to that published in the International Journal of Bifurcation and Chaos (2018), https://doi.org/10.1142/S021812741830001X

${ }^{\dagger}$ Departament de Matemàtiques (Universitat Politècnica de Catalunya), Av. Gregorio Marañón 44-50, 08028 Barcelona

${ }^{\ddagger}$ Department of Mathematics (KTH, Royal Institute of Technology), SE-100 44 Stockholm

$\S$ Departament de Matemàtiques (Universitat Politècnica de Catalunya), Av. Gregorio Marañón 44-50, 08028 Barcelona; and Barcelona Graduate School of Mathematics (BGSMath), Campus de Bellaterra, Edifici C, 08193 Bellaterra, Spain

IDepartament de Matemàtiques i Informàtica (Universitat de Barcelona), Gran Via de les Corts Catalanes 585, 08007 Barcelona; and Barcelona Graduate School of Mathematics (BGSMath), Campus de Bellaterra, Edifici C, 08193 Bellaterra, Spain. Corresponding author: fontich@ub.edu

" Centre de Recerca Matemàtica, Campus de Bellaterra, Edifici C, 08193 Bellaterra, Spain; and Barcelona Graduate School of Mathematics (BGSMath), Campus de Bellaterra, Edifici C, 08193 Bellaterra, Spain. Corresponding author: E-mail: jsardanyes@crm.cat 
gap. The implications of this phenomenon are discussed in the context of delayed transitions associated to the so-called saddle remnants.

Keywords: Bifurcations; Hypercycles; Origins of life; Complex systems; Periodic orbits.

\section{Introduction}

The detailed characterization of bifurcations in nonlinear systems constitutes a very important issue to understand qualitative changes in dynamics. Mathematical investigations of cooperative systems (e.g., molecular species with catalytic interactions) have been carried out using different modeling approaches, the hypercycle model being one of the most important ones. Hypercycles are catalytic sets of macromolecules that can catalyze their own replication or the replication of other species of the network, which have a cyclic architecture [Eigen \& Schuster, 1979]. Hypercycles have been largely investigated in the framework of prebiotic evolution [Eigen, 1971;Eigen \& Schuster, 1979; Campos et al., 2000; Hofbauer et al., 1990; Smith \& Szathmáry, 1995].

The shift from survival to extinction in hypercycles is governed by bifurcations. The analysis of bifurcations in hypercycles has been classically restricted to low dimensional time-continuous systems, specially for so-called symmetric hypercycles [Nuño et al., 1993; Silvestre \& Fontanari, 2008], for which all of the species have the same kinetic properties (i.e., they are considered neutral mutants [Silvestre \& Fontanari, 2008]). Few works have explored asymmetries in hypercycles: in two-member cycles [Sardanyés \& Solé, 2006] or in larger hypercycles by means of numerical results [Campos et al., 2000]. Moreover, few studies have focused on the dynamics of large hypercycles [Silvestre \& Fontanari, 2008], and especially, in the bifurcations found in hypercycles with $n \geq 5$ species. It is known that, under appropriate parameter values, hypercycles are bistable systems. Under bistability, the asymptotic coexistence or extinction of hypercycles depends on the initial conditions. It is also known that the nature of the coexistence attractor largely depends on the dimension (number of catalytic species) of the hypercycle. The bifurcations for this type of systems are mainly given by saddle-node (hereafter s-n) bifurcations, which involve a catastrophic (i.e., sharp) extinction as the bifurcation parameters crosses its bifurcation value [Sardanyés \& Solé, 2006; Sardanyés \& Solé, 2007; Silvestre \& Fontanari, 2008].

The detailed mechanisms responsible for bifurcations in hypercycles have been provided by several authors [Sardanyés \& Solé, 2006; Sardanyés \& Solé, 2007; Nuño et al., 1993]. For instance, Silvestre \& Fontanari [2008] showed that the conditions of viability for symmetric hypercycles competing with an error-tail hold for all $n$, not only when fixed points were stable. That is because they found numerically that the viability condition of hypercycle was the same as the one that guarantees the existence of real fixed points. In particular, they considered a symmetric hypercycle with $n=12$ members, modeled with the 
system equations:

$$
\left\{\begin{array}{l}
\dot{x}_{i}=x_{i}\left(A_{i} Q+k_{i} x_{i-1} Q-\phi\right), \\
\dot{x}_{e}=x_{e}\left(A_{e}-\phi\right)+(1-Q) \sum_{i=1}^{n} x_{i}\left(A_{i}+k_{i} x_{i-1}\right),
\end{array}\right.
$$

describing the time evolution of the $i$ th hypercycle species $\left(x_{i}\right)$ and the error tail $\left(x_{e}\right)$. Considering catalytic constants $k_{i}=1$, for all $i$, and $A=10^{-3}$ (Malthusian replication constant), there is a nonzero equilibrium point if and only if $Q \gtrsim 0.19639, Q$ being the copying fidelity during replication (see Section 2 for a detailed description of the model). They found numerically that only when $Q \gtrsim 0.19639$ there exists a stable periodic solution and so the hypercycle is viable. Therefore, the existence of an unstable fixed point seemed to be a necessary condition for the presence of stable periodic orbits in a hypercycle.

More recently, Guillamon et al., [2015] investigated the periodic orbits in symmetric hypercycles with $n=5$. In particular, they studied how these orbits behave in terms of $Q$ using both numerical and analytical methods. As an example, the results they obtained using $A=0.5$ were the value $Q_{S S}=0.91607$, computed analytically, at which two unstable fixed points undergo a s-n bifurcation and the value of $Q_{P O}=0.91614$, computed numerically, at which a s-n bifurcation of periodic orbits takes place, thus causing the asymptotic extinction of the system for any initial condition. Interestingly, and contrary to what Silvestre \& Fontanari [2008] observed, the two values were not exactly the same. Therefore, a slight gap between the two s-n bifurcations was found (see Figure $1)$.

In this manuscript we aim at exploring the effects of two important features of hypercycle models on the persistence and characteristics of the bifurcation gaps previously reported. First, we analyse the impact of asymmetries in Malthusian replication constants in the bifurcation gap. Asymmetric hypercycles are more biologically-realistic systems since symmetric ones consider that the templates forming the hypercycle are neutral mutants [Silvestre \& Fontanari, 2008]. However, it is known that mutations have differential fitness effects [Sanjuán et al., 2004; Carrasco et al., 2007]. That is, mutants can carry deleterious, neutral, lethal, or beneficial mutations. Second, we explore the impact of the size of the hypercycle on this bifurcation gap, focusing on symmetric hypercycles. Moreover, the analyses of bifurcations in larger hypercycles becomes relevant since, albeit being much more susceptible to stochastic extinctions [Kauffman, 1993], they could store more information.

The paper is organized as follows. In Section 2 we introduce the mathematical model analysed, summarizing its dynamics. In Section 3.1, we explore asymmetric hypercycles for $n=5$, the lowest dimension with the presence of periodic orbits. Within this section we first perform an analytical study of an $n$-component asymmetric hypercycle, following the work conducted in Guillamon et al., [2015] for symmetric hypercycles. We later concentrate on the 5 -component asymmetric hypercycles. This study allows to know precisely the locus of the s-n bifurcations of equilibria, namely $Q=Q_{S S}$. Then, a numerical study of the 5-component asymmetric hypercycle is made to carry out the 

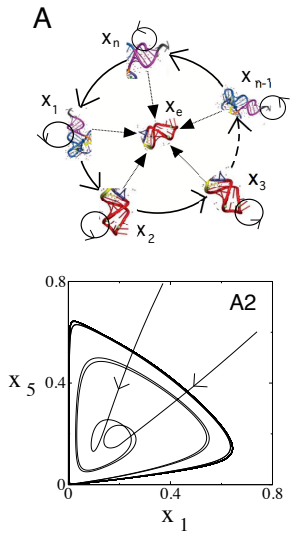

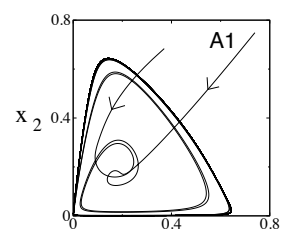

$\mathrm{X}_{1}$

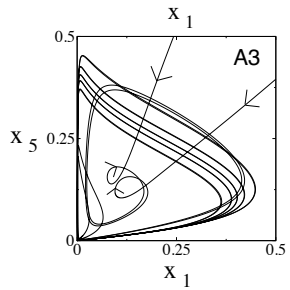

B
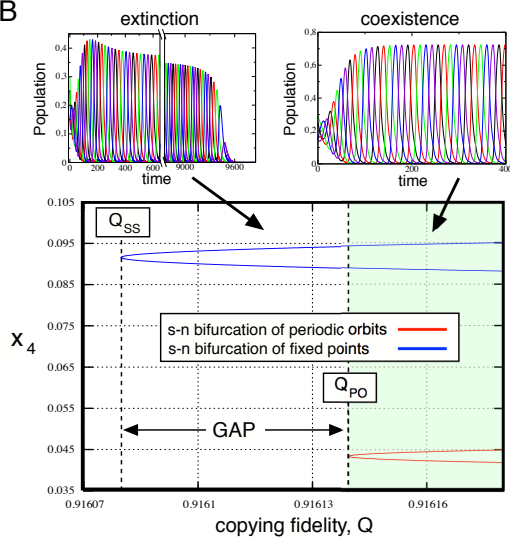

Figure 1: (A) Hypercycle formed by $n$ species. The phase portraits display several projections of the periodic orbits allowing coexistence of species (A1 and A2). Panel A3 displays the same projection than in panel A2 but with parameters after the saddle-node (s-n) bifurcation of periodic orbits. Under this scenario, the flows display transient periodic behavior since the trivial attractor is asymptotically globally stable. (B) Bifurcation curves of periodic orbits (red) and of fixed points (blue) taking coordinate $x_{4}$ (see [Guillamon et al., 2015] for details) with respect to parameter $Q$ for the symmetric hypercycle, i.e., $\delta=0$, with $A=0.5$ and $k=1$. The parametric region in green allows for the coexistence of the hypercycle members in an oscillatory regime. The bifurcation value of periodic orbits, $Q_{P O}$, and of the fixed points, $Q_{S S}$, are indicated with dashed vertical lines. Notice that both bifurcation values do not coincide, fact which has been denoted as a bifurcation gap [Guillamon et al., 2015]. The smaller panels display time series for all hypercycle members in the extinction and coexistence scenarios. 
bifurcation analysis. Finally, in Section 3.2. we explore the behavior of the bifurcation gap for larger hypercycles, focusing on the cases with $n=6$ and $n=8$ catalytic species.

\section{Mathematical model}

We analyze the hypercycle model introduced by Campos et al., [2000], which describes the dynamics of an $n$-member hypercycle competing with its error tail (see Fig. 1A for a schematic diagram). The model is given by the next set of ordinary differential equations:

$$
\left\{\begin{array}{l}
\dot{x}_{i}=x_{i}\left(A_{i} Q+k_{i} x_{i-1} Q-\phi\right), \\
\dot{x}_{e}=x_{e}\left(A_{e}-\phi\right)+(1-Q) \sum_{i=1}^{n} x_{i}\left(A_{i}+k_{i} x_{i-1}\right),
\end{array}\right.
$$

with $i=1, \ldots, n$ and $\phi=\sum_{i=1}^{n} x_{i}\left(A_{i}+k_{i} x_{i-1}\right)+A_{e} x_{e}$. State variable $x_{i}$ is the concentration of the $i$ th template, $x_{e}$ being the concentration of the mutant replicators. To introduce the cyclic architecture of catalysts we set $x_{0}=x_{n}$. Parameter $A_{i} \in(0,1]$ is the replication rate of species $i, k_{i}$ is the strength of the catalysis that $x_{i-1}$ has on $x_{i}$ replication, and $Q \in(0,1]$ is the copying fidelity of the templates during replication. Concerning the mutant species, $A_{e} \in(0,1]$ is their replication rate. Finally, $\phi$ is a dilution flow that keeps the total population constant also introducing competition between all of the replicators. Since the structure of the equations determine that $\sum_{i=1}^{n} x_{i}+x_{e}=1$ is invariant, we consider the system in this domain and hence we can forget about the population of mutants, $x_{e}$.

A hypercycle is called symmetric if $A_{i}=A_{e}=A$ and $k_{i}=k$ for all $i$, and asymmetric otherwise. Looking at Eq. (1), it is clear that a symmetric structure eases the computations. Let us summarize some important properties of system (1) in the symmetric case, see also [Campos et al., 2000; Silvestre \& Fontanari, 2008; Guillamon et al., 2015]:

1. It is a bistable system for some set of parameters. The origin is always an attractor. When $n \leq 4$, there is bistability when $Q>Q_{S S}$ and the coexistence attractor - which allows the persistence of all the hypercycle members - is a non trivial equilibrium point. When $n \geq 5$, there is bistability as well for $Q>Q_{P O}$, and the coexistence attractor is a periodic orbit.

2. For $n=5, Q_{P O}$ and $Q_{S S}$ do not coincide. Then, there is a bifurcation gap in the parameter space where two non-trivial equilibria exist but periodic orbits do not [Guillamon et al., 2015].

In this paper we break the symmetry by making parameters $A_{i}$ to be heterogeneous up to some extent, while keeping $k_{i}=1$ for all $i=1, \ldots, n$. In other words, here we focus on asymmetries in the so-called Malthusian replication rates, given by the exponential replication (at low population numbers) of the hypercycle species, keeping symmetries in the non-linear replication terms given 
by heterocatalysis. More precisely, we will consider cases in which $A_{i}=A$ for all $i=1, \ldots, n$ except for one or two $i$ values for which $A_{i}=a=A-\delta$, where $\delta$ (with $\delta<A$ ) will be considered as the asymmetry parameter in Malthusian replication and $a, A \in(0,1]$. In particular, we will mainly deal with three cases:

- Case $a A \ldots A$ : $A_{1}=a, A_{e}=A$ and $A_{i}=A$ for all $i=2, \ldots, n$.

- Case aaA...A: $A_{1}=A_{2}=a, A_{e}=A$ and $A_{i}=A$ for all $i=3, \ldots, n$.

- Case aAa...A: $A_{1}=A_{3}=a, A_{e}=A$ and $A_{i}=A$ for all $i=2,4, \ldots, n$.

Given the cyclic structure of (1), these cases represent many other cases with one or two different values.

Finally, we consider the effect of increasing the dimension (size) of the hypercycle, focusing on the symmetric case. Concretely we deal with $n=5,6,8$.

\section{Results and discussion}

\subsection{Asymmetric hypercycles}

\subsection{1 n-member hypercycle}

We start studying analytically a general asymmetric case with $n$ templates, looking for general properties of asymmetric hypercycles. In the following we are going to consider the system

$$
\dot{x}_{i}=x_{i}\left(A_{i} Q+x_{i-1} Q-\phi\right), \quad i=1, \ldots, n,
$$

for the different cases above mentioned, with $\phi=\sum_{i=1}^{n} x_{i}\left(A_{i}+x_{i-1}\right)+A_{e}(1-$ $\left.\sum_{i=1}^{n} x_{i}\right)$ and $x_{0}=x_{n}$. We recall that we do not take into account the equation for $x_{e}$ since $\sum_{i=1}^{n} x_{i}+x_{e}=1$.

We are interested in studying the fixed points of system (2), its saddlenode ( $\mathrm{s}-\mathrm{n}$ ) bifurcation and the regions where periodic orbits can be found, in a way analogous to the one conducted in [Guillamon et al., 2015]. To simplify notation we will also refer to the right-hand side of $(2)$ as the vector field $F(x)$. Our analysis will consist of two steps. In the first one, we find fixed points and the $s-n$ bifurcation of fixed points. In the second step, we determine a region in which we can find periodic orbits, i.e., where coexistence dynamics holds. For that we analytically compute regions where it is impossible for periodic orbits to exist.

Fixed points. We first deal with the case $a A \ldots A$, which is the simplest asymmetric structure one can consider. It is clear that $(0, \ldots, 0)$ is always an equilibrium point. There are also fixed points for which some of their coordinates are 0 , but we do not compute them here since there is a huge number of possible 
combinations and knowing them is not necessary for the study we want to conduct. Imposing $x_{i} \neq 0$ for all $i$ in Eq. (2) we get

$$
\left\{\begin{array}{l}
(A-\delta) Q+x_{n} Q-\phi=0, \\
A Q+x_{1} Q-\phi=0 \\
\vdots \\
A Q+x_{n-1} Q-\phi=0
\end{array}\right.
$$

which results in

$$
\left\{\begin{array}{l}
x_{n}=\frac{\phi-(A-\delta) Q}{Q} \\
x_{1}=\cdots=x_{n-1}=\frac{\phi-A Q}{Q} .
\end{array}\right.
$$

Subtracting $x_{1}$ to $x_{n}$, we get $x_{n}-x_{1}=\delta$.

Thus, we only need to find the expression of one of the $x_{i}$. Let us find $x_{1}$. We first express $\phi$ in terms of $x_{1}$ :

$$
\begin{aligned}
\phi & =\sum_{i=1}^{n} x_{i}\left(A_{i}+x_{i-1}\right)+x_{e} A=-\delta x_{1}+A+\sum_{i=1}^{n} x_{i} x_{i-1} \\
& =\delta x_{1}+A+n x_{1}^{2},
\end{aligned}
$$

and substituting it into the $x_{1}$ expression above we get

$$
n x_{1}^{2}-(Q-\delta) x_{1}+A(1-Q)=0 .
$$

Therefore, there are two equilibrium points of the form

$$
\left(x_{ \pm}, \ldots, x_{ \pm}, x_{ \pm}+\delta\right)
$$

with $x_{ \pm}=\frac{(Q-\delta) \pm \sqrt{(Q-\delta)^{2}-4 n A(1-Q)}}{2 n}$.

From this last expression we know that there is a s-n bifurcation occurring for a value of $Q$ for which

$$
(Q-\delta)^{2}-4 n A(1-Q)=0,
$$

that is

$$
Q=Q_{S S}=2(\sqrt{n A(1+n A-\delta)}-n A)+\delta>\delta .
$$

The two equilibrium points borning at the s-n bifurcation at $Q=Q_{S S}$ exist for $Q>Q_{S S}$. The bifurcation point is therefore:

$$
\left(\frac{Q_{S S}-\delta}{2 n}, \ldots, \frac{Q_{S S}-\delta}{2 n}, \frac{Q_{S S}-\delta}{2 n}+\delta\right) .
$$

In the symmetric case this point was $\left(\frac{Q}{2 n}, \ldots, \frac{Q}{2 n}, \frac{Q}{2 n}\right)$, with $Q=\left.Q_{S S}\right|_{\delta=0}$, so that the bifurcation point for the asymmetric case tends to the one for the symmetric case when $\delta$ tends to zero. 
(a)

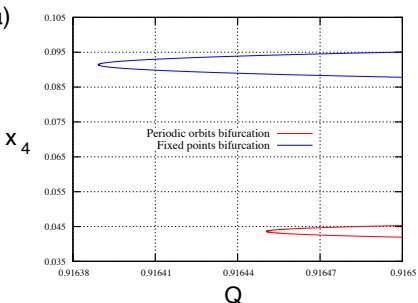

(c)

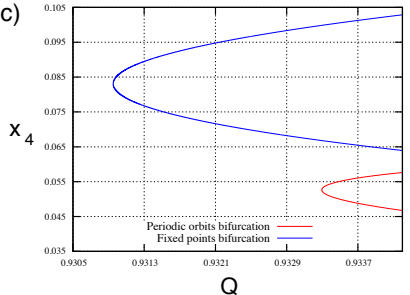

(b)

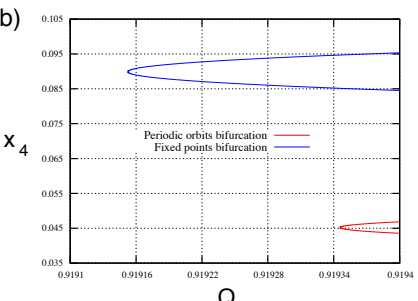

(d)

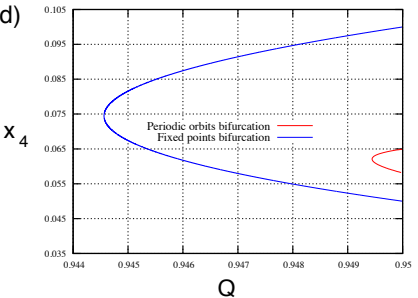

Figure 2: Bifurcation diagrams for 5-member asymmetric hypercycles with structure $a a A A A$ setting $A=0.5$ and: $\delta=0.001$ (a), 0.01 (b), 0.05 (c) and 0.1 (d). The saddle-node (s-n) bifurcation of periodic orbits is indicated with red curves, while the s-n bifurcation of fixed points is indicated in blue.

Now that we have conducted a brief study of the simplest $n$-dimensional asymmetric hypercycle, we study hypercycles where two of the templates have a replication rate $a=A-\delta$. In particular we are going to consider cases $a a A \ldots A$ and $a A a A \ldots A$, since these are the two cases we will focus on lately when dealing with 5 -member asymmetric hypercycles. In the case of 5 members, these two cases cover all possibilities when two different values of $A_{i}$ are considered (relabelling the templates, if necessary). The procedure to obtain the equilibrium points is similar to the above case $a A \ldots A$. We obtain

$$
\begin{array}{ll}
\left(x_{ \pm}+\delta, x_{ \pm}, \ldots, x_{ \pm}, x_{ \pm}+\delta\right), & \text { for case } a a A \ldots A ; \\
\left(x_{ \pm}, x_{ \pm}+\delta, \ldots, x_{ \pm}, x_{ \pm}+\delta\right), & \text { for case } a A a A \ldots A,
\end{array}
$$

where $x_{ \pm}=\frac{Q-2 \delta \pm \sqrt{(Q-2 \delta)^{2}-4 n A(1-Q)}}{2 n}$. Now the bifurcation value is

$$
Q=Q_{S S}^{\prime}=2(\sqrt{n A(1+n A-2 \delta)}-n A)+2 \delta>2 \delta,
$$

in both cases and the bifurcation points are

$$
\left(\frac{Q_{S S}^{\prime}-2 \delta}{2 n}+\delta, \frac{Q_{S S}^{\prime}-2 \delta}{2 n}, \ldots, \frac{Q_{S S}^{\prime}-2 \delta}{2 n}, \frac{Q_{S S}^{\prime}-2 \delta}{2 n}+\delta\right),
$$

for case $a a A \ldots A$;

$$
\left(\frac{Q_{S S}^{\prime}-2 \delta}{2 n}, \frac{Q_{S S}^{\prime}-2 \delta}{2 n}+\delta, \ldots, \frac{Q_{S S}^{\prime}-2 \delta}{2 n}, \frac{Q_{S S}^{\prime}-2 \delta}{2 n}+\delta\right),
$$


for case $a A a A \ldots A$.

Periodic orbits. Since our main goal here is to study the behaviour of periodic orbits with respect to the parameter $Q$, we first care about the conditions for their existence. In particular we are going to locate a subset of the basin of attraction of the origin, and consider the complementary of this region as the domain where the existence of periodic orbits is possible depending on $Q$. For this purpose, we define the domains $\Omega_{\alpha}$ and the hyperplanes $\Sigma_{\alpha}$ as:

$$
\begin{aligned}
\Omega_{\alpha} & :=\left\{x \in \mathbb{R}^{n} \mid x_{i} \geq 0, \sum_{i=1}^{n} x_{i} \leq \alpha\right\} \\
\Sigma_{\alpha} & :=\left\{x \in \mathbb{R}^{n} \mid \sum_{i=1}^{n} x_{i}=\alpha\right\}
\end{aligned}
$$

for $\alpha \in(0,1]$. Observe that the dynamics of system (2) is restricted to $\Omega_{1-x_{e}}$ which is a subset on the $n$-simplex $\Omega:=\Omega_{1}$. Besides, each subspace generated by vectors of the canonical basis is invariant; in particular, the hyperplanes $x_{i}=0$ are invariant. Thus, except for $\Sigma_{\alpha}$, all other $n$ faces of $\Omega_{\alpha}$, for $\alpha \in(0,1]$, are invariant for the vector field $F$. We study the flow generated by $F$ on $\Sigma_{\alpha}$ by taking the vector $v=(1, \ldots, 1)$, which is normal to the hyperplane, and computing $v \cdot F(x)$ for $x \in \Sigma_{\alpha}$. We have that

$$
\cos (v, F(x))=\frac{v \cdot F(x)}{\|v\|\|F(x)\|} .
$$

Then, when this scalar product is zero, $F$ is tangent to the hyperplane. When it is positive, $F$ points outside of $\Omega_{\alpha}$, and when it is negative $F$ points inside $\Omega_{\alpha}$. If $F$ points inside $\Omega_{\alpha}$, for all points in $\Sigma_{\alpha}$, we have that $\Omega_{\alpha}$ is positively invariant, which means that no orbits escape from $\Omega_{\alpha}$ in forward time. We also know that if the vector field $F$ crosses transversally $\Sigma_{\alpha}$ for all $\alpha<\alpha_{0}$ for some $\alpha_{0}$, then $\Omega_{\alpha_{0}}$ is contained in the basin of attraction of the origin. Thus, periodic orbits can only exist in the complementary of the region where this occurs.

This setting is general for any case of asymmetry, but the determination of the suitable values of $\alpha$ that give information about the location of periodic orbits must be performed case by case. As a general example, in this section we study the case $a A \ldots A$. Next, in Section 3.1.2, we will apply the same procedure to 5 -member hypercycles with two asymmetries. For the case $a A \ldots A$, the numerator of $\cos (v, F(x))$ becomes

$$
\begin{aligned}
v \cdot F\left(x_{1}, \ldots, x_{n}\right)= & (Q-\alpha)\left(\sum_{i=1}^{n} x_{i-1} x_{i}-\delta x_{1}\right) \\
& +A \alpha(Q-1) .
\end{aligned}
$$

To study the behavior of expression (6) for a given $\alpha<1$, we distinguish two cases. If $Q \leq \alpha$, the product (6) is negative (we use $\delta<A$ ) so $F$ points inside $\Omega_{\alpha}$ and thus $\Omega_{\alpha}$ is positively invariant. If $Q>\alpha$, we need the maximum of 
(a)

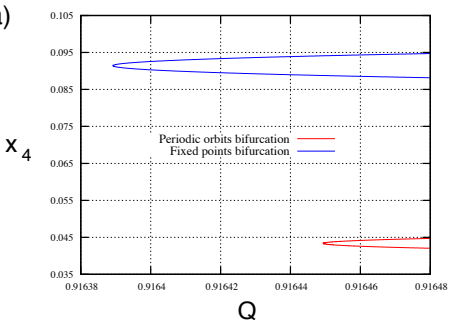

(c)

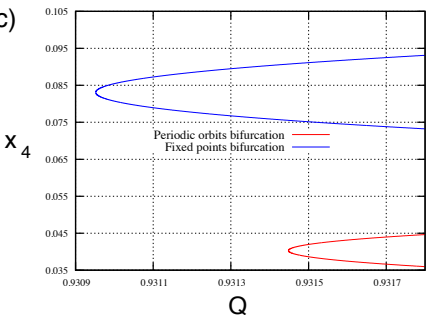

(b)

$\mathrm{X}_{4}$

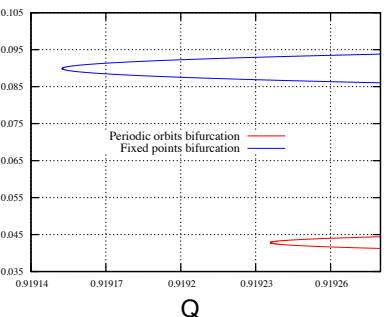

(d)

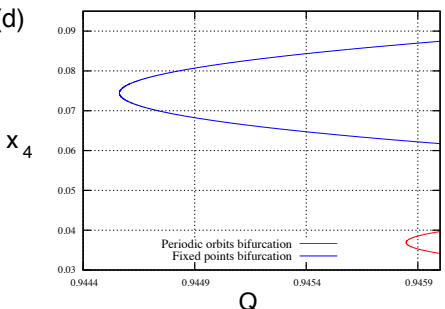

Figure 3: Same as Fig. 2 for 5-member asymmetric hypercycles with structure $a A a A A$ setting $A=0.5$ and: $\delta=0.001$ (a), 0.01 (b), 0.05 (c) and 0.1 (d).

the RHS of (6). We first maximize $f(x)=\sum_{i=1}^{n} x_{i-1} x_{i}$, using the method of Lagrange multipliers. We want:

$$
\max _{x} \sum_{i=1}^{n} x_{i-1} x_{i}, \quad \text { subject to } \quad \sum_{i=1}^{n} x_{i}=\alpha,
$$

together with $x_{i} \geq 0$. We obtain a unique critical point: $x^{*}=\left(\frac{\alpha}{n}, \ldots, \frac{\alpha}{n}\right) \in \Sigma_{\alpha}$ and $f\left(x^{*}\right)=\frac{\alpha^{2}}{n}$. We need to check whether the maximum reached on the boundary of $\Sigma_{\alpha}$ is greater than the value we have found.

When $n=2$, the function $f(x)=x_{1} x_{2}$ is always 0 on the boundary of $\Sigma_{\alpha}$. Hence, the maximum is reached in the interior and turns out to be $\alpha^{2} / 2$.

When $n=3$, the function $\sum_{i=1}^{3} x_{i-1} x_{i}$ is nonzero on the boundary when only one $x_{i}$ is 0 . Due to the cyclic structure of the previous function, it is indistinct which coordinate we choose to be 0 . Assuming $x_{3}=0$, we want:

$$
\max _{x} x_{1} x_{2}, \quad \text { subject to } \quad x_{1}+x_{2}=\alpha, \quad x_{i} \geq 0 .
$$

Using the method of Lagrange multipliers we get that the maximum is $\alpha^{2} / 4$. Thus, the maximum on the boundary is less than the maximum that is achieved in the interior of $\Omega_{\alpha}$, which is $\alpha^{2} / 3$. When $n=4$, in the boundary of $\Sigma_{\alpha}$ either one, two, or three of the $x_{i}$ may vanish. If three of them vanish, $f$ is zero. If two of them vanish, they can be consecutive and then we get a problem equivalent 
to (8) for which the maximum is $\alpha^{2} / 4$, or non-consecutive and then the function is 0 . If only one of the terms vanishes, for instance $x_{4}$, we have to consider

$$
\max _{x}\left(x_{1} x_{2}+x_{2} x_{3}\right), \text { subject to } x_{1}+x_{2}+x_{3}=\alpha, \quad x_{i} \geq 0 .
$$

Using again the method of Lagrange multipliers we get that $\alpha^{2} / 4$ is the maximum, which coincides with the maximum reached in the interior of $\Omega_{\alpha}$. Therefore, the maximum is attained both on the boundary and in its interior.

When $n>4$, we can proceed similarly. When two or more $x_{i}$ vanish, we obtain a problem equivalent to the one solved for a lower $n$. We only need to solve a "new" optimization problem when only one of the $x_{i}$ is 0 . We are going to solve it by induction.

Let us see now that $\forall n>4$ the maximum of (7) is $\alpha^{2} / 4$, attained on the boundary when one and just one of the $x_{i}$ is 0 . As the objective function has a cyclic structure, it does not matter which of the $x_{i}$ is picked up to be 0 . Let us suppose, by induction, that the maximum $\alpha^{2} / 4$ holds for $n-1$ replicators. Then we take $n$ replicators. The image of the critical point is $\frac{\alpha^{2}}{n}$. We want to solve (7) on the boundary. Taking $x_{n}=0$, we have:

$$
f(x)=x_{1} x_{2}+\cdots+x_{n-2} x_{n-1} .
$$

Now, as $x_{i}>0, i=1, \ldots, n-1$,

$$
f(x) \leq x_{1} x_{2}+\cdots+x_{n-2} x_{n-1}+x_{n-1} x_{1},
$$

the RHS being the function we have for $n-1$ replicators. Hence, from the induction hypothesis, the maximum of this function is $\alpha^{2} / 4$. Therefore, $\forall n \geq 4$, $\sum_{i=1}^{n} x_{i-1} x_{i} \leq \frac{\alpha^{2}}{4}$, for $\sum_{i=1}^{n} x_{i}=\alpha, x_{i} \geq 0$.

Moreover, for $n \geq 4, \sum_{i=1}^{n} x_{i-1} x_{i}-\delta x_{1} \leq \alpha^{2} / 4$, and the maximum $\alpha^{2} / 4$ is attained at $x_{2}=x_{3}=\alpha / 2, x_{1}=x_{j}=0,4 \leq j \leq n$. Then we get

$$
v \cdot F\left(x_{1}, \ldots, x_{n}\right) \leq(Q-\alpha) \frac{\alpha^{2}}{4}+A \alpha(Q-1) .
$$

We are going to find a threshold for $Q$, denoted by $Q_{*}$, such that $\forall Q \geq Q_{*}$, the region $\Omega_{\alpha}$ is positively invariant.

We can find the zeros of the RHS of (9), which are $\alpha_{0}=0$ and

$$
\alpha_{ \pm}=\frac{Q \pm \sqrt{Q^{2}-16 A(1-Q)}}{2} .
$$

Looking for which $Q$ expression (10) is real we get the threshold:

$$
Q_{*}=-8 A+4 \sqrt{A(4 A+1)} .
$$

Therefore, if $Q \geq Q_{*}$, then $v \cdot F\left(x_{1}, \ldots, x_{n}\right)<0$ for $\alpha \in\left(0, \alpha_{-}\right) \cup\left(\alpha_{+}, Q\right)$. This means that when $\alpha<\alpha_{-}$or $\alpha>\alpha_{+}, \Omega_{\alpha}$ is positively invariant. Since for 


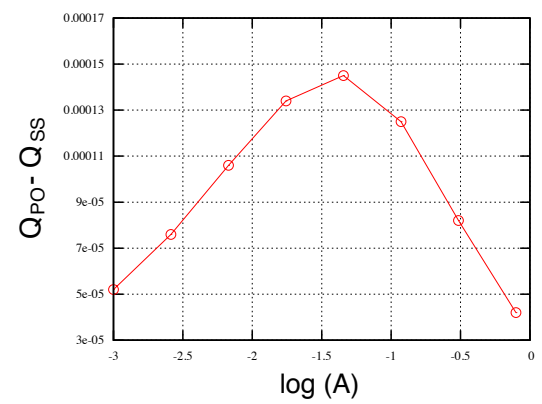

Figure 4: Measure of the gap size between the two bifurcations for different values of $A$, in the symmetric case (see [Guillamon et al., 2015] for further details).

all $\alpha<\alpha_{-}, v \cdot F\left(x_{1}, \ldots, x_{n}\right)<0$, for initial conditions in $\Omega_{\alpha}$ with $\alpha<\alpha_{-}$, the total population of the hypercycle tends to 0 . Moreover, in order to look for periodic orbits or other recurrent phenomena we have to concentrate on values of $\alpha \in\left(\alpha_{-}, \alpha_{+}\right)$and $Q \geq Q_{*}$.

We need to note that this interval is not sharp because the inequality (9) is not sharp. Hence, for values of $\alpha$ in the interval and close to the interval limits, $\Omega_{\alpha}$ is possibly still positively invariant so, to be sure to truly find interesting behaviour, we should choose values of $\alpha$ close to the midpoint of the interval.

Now, if the discriminant of (10) was to be negative, that is, if $Q<Q_{*}$, then $\alpha_{-}$and $\alpha_{+}$are complex and the only real zero is $\alpha_{0}=0$. Therefore, as the RHS of (9) tends to $-\infty$ when $\alpha$ grows, it is always less or equal than zero. Then, $\Omega_{\alpha}$ is positively invariant $\forall \alpha$, which means that the origin is globally asymptotically stable.

Summarizing, consider the hypercycle formed by $n$ replicators with Malthusian replication rates $a A \ldots A$, and let $\Omega_{\alpha}$ be given by (4) and $Q_{*}$ be given by (11). Then,

- when $\alpha \geq Q, \Omega_{\alpha}$ is positively invariant. Moreover, since $v \cdot F<0$ in $\left\{x \in \Omega \mid x_{1}+\cdots+x_{n}>Q\right\}$, there can not be periodic orbits in that domain;

- when $\alpha<Q$,

- if $Q<Q_{*}, \Omega_{\alpha}$ is positively invariant, and $v \cdot F<0$ in $\Omega$. Then, there can not be periodic orbits in $\Omega$;

- if $Q \geq Q_{*}, \Omega_{\alpha}$ is positively invariant for $\alpha \in\left(0, \alpha_{-}\right) \cup\left(\alpha_{+}, Q\right)$, where $\alpha_{+}$and $\alpha_{-}$are given by (10). Moreover, $v \cdot F<0$ in $\{x \in \Omega \mid$ $\left.x_{1}+\cdots+x_{n}<\alpha_{-}\right\} \cup\left\{x \in \Omega \mid x_{1}+\cdots+x_{n}>\alpha_{+}\right\}$. Then, if periodic orbits exist, for each point of the periodic orbit the sum of its coordinates must be between $\alpha_{-}$and $\alpha_{+}$. 
(a)

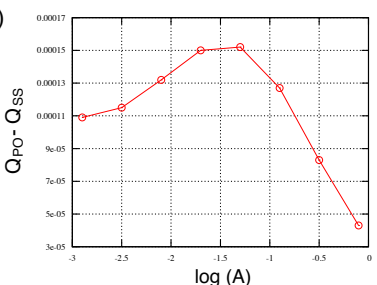

(c)

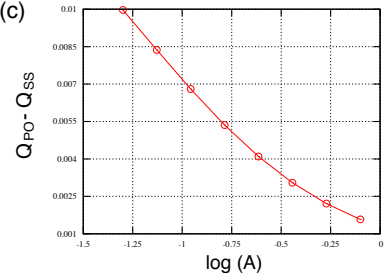

(b)

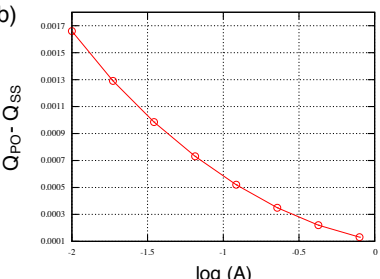

$\log (A)$

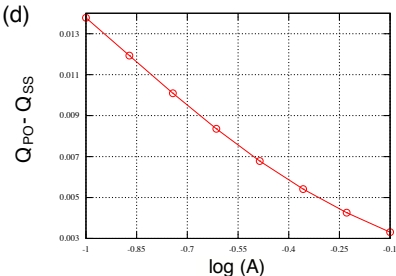

Figure 5: Bifurcation gap for the asymmetric case $a a A A A$ with: $\delta=0.001$ (a), 0.01 (b), 0.05 (c) and 0.1 (d).

Hence, we have located, depending on the parameter $Q$, the regions where periodic orbits can appear. In order to find them explicitly, we need to resort to numerical methods, see Section 5.3. in the Appendix.

Next, we apply the same procedure to complete all possible asymmetric cases with two different replication rates for dimension $n=5$, which is a tradeoff between analytical tractability of equations and existence of periodic orbits [Silvestre \& Fontanari, 2008]. In particular, as commented above, all of the remaining cases are $a a A A A$ and $a A a A A$.

\subsubsection{Five-member hypercycle}

From (3), we know that the equilibrium points are $\left(x_{ \pm}+\delta, x_{ \pm}, x_{ \pm}, x_{ \pm}, x_{ \pm}+\delta\right)$ for the case $a a A A A$, and $\left(x_{ \pm}, x_{ \pm}+\delta, x_{ \pm}, x_{ \pm}, x_{ \pm}+\delta\right)$ for the case $a A a A A$, where

$$
x_{ \pm}=\left((Q-2 \delta) \pm \sqrt{(Q-2 \delta)^{2}-20 A(1-Q)}\right) / 10 .
$$

As $x_{ \pm}$is the same in both cases, the critical value of $Q$ for which there is a s-n bifurcation of fixed points, $Q_{S S}$, is the same:

$$
Q_{S S}=2(\sqrt{5 A(1+5 A-2 \delta)}-5 A+\delta) .
$$

Therefore, the respective bifurcation points can also be obtained from (12) with $Q=Q_{S S}$.

As our goal is to study both the bifurcations of fixed points and of periodic orbits in these two cases, let us find the regions of $\Omega$ where periodic orbits can be found. We perform the analogous procedure explained in Section 3.1.1 for the case $a A \ldots A$ to obtain the corresponding regions in the present cases. We provide the results next. 
We need to study the sign of $v \cdot F(x)$ which becomes

$$
(Q-\alpha)\left(\sum_{i=1}^{5} x_{i} x_{i-1}-\delta\left(x_{1}+x_{2}\right)\right)+A \alpha(Q-1),
$$

for the $a a A A A$ case, and

$$
(Q-\alpha)\left(\sum_{i=1}^{5} x_{i} x_{i-1}-\delta\left(x_{1}+x_{3}\right)\right)+A \alpha(Q-1),
$$

for the $a A a A A$ case. In both cases, given

$$
\begin{aligned}
& \alpha_{ \pm}=\frac{Q+ \pm \sqrt{Q^{2}+16 A(Q-1)}}{2}, \\
& Q_{*}=-8 A+4 \sqrt{4 A^{2}+A}
\end{aligned}
$$

and adapting the calculations done in Section 3.1.1, if periodic orbits exist, they must lie in the region of the parameter space where $Q \geq Q_{*}$ and, moreover, the sum of the coordinates of its points, $\alpha$, fulfill $\alpha>Q$ and $\alpha \in\left[\alpha_{-}, \alpha_{+}\right]$.

From this point on, we need to implement numerical routines (see Section 5.1. in the Appendix), in order to find periodic orbits of system (2) for the cases $a a A A A$ and $a A a A A$ and, in particular, the parameter values of the corresponding s-n bifurcations of periodic orbits, $Q_{P O}$. An important issue is the choice of a Poincaré section. Adapting (3) to the case $n=5$, we know that the equilibrium points are $(x+\delta, x, x, x, x+\delta)$ for the case $a a A A A$, and $(x, x+\delta, x, x, x+\delta)$ for the case $a A a A A$, with $x=\left((Q-2 \delta) \pm \sqrt{(Q-2 \delta)^{2}-20 A(1-Q)}\right) / 10$. Clearly, the average copying fidelity rate at which the s-n bifurcation of fixed points occurs is:

$$
Q_{S S}=2(\sqrt{5 A(1+5 A-2 \delta)}-5 A+\delta) .
$$

Keeping in mind that our goal is to find periodic orbits, it is clear that the Poincaré section should not be close to equilibrium points. A natural choice is to fix one of the coordinates, for instance $x_{1}$, at the value corresponding to the first coordinate of the equilibrium point at the bifurcation $Q=Q_{S S}$. Then, if $Q-Q_{S S}$ is not too small, the Poincare section $\left(\Sigma_{P}\right)$ will be far enough from both of them. Accordingly, we consider

$$
\Sigma_{P}=\left\{x_{1}=\frac{Q-2 \delta}{10}+\delta\right\}
$$

for the $a a A A A$ case, and

$$
\Sigma_{P}=\left\{x_{1}=\frac{Q-2 \delta}{10}\right\}
$$

for the $a A a A A$ case. This choice is crucial for the success of the numerical continuation with respect to parameters. Taking, for instance, the section given 
(a)

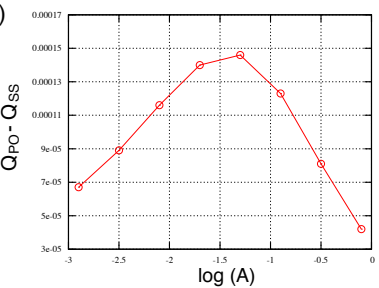

(c)

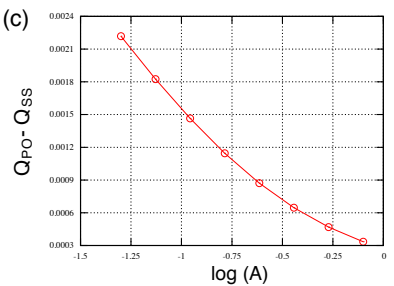

(b)

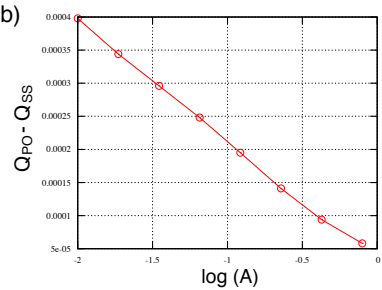

$\log (A)$

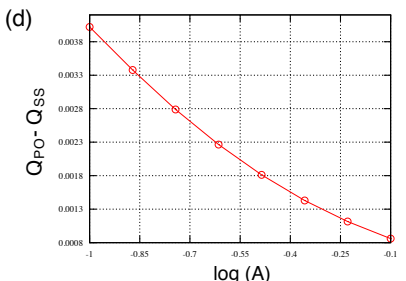

Figure 6: Same as Fig. 5 for case $a A a A A$ with: $\delta=0.001$ (a), 0.01 (b), 0.05 (c) and $0.1(\mathrm{~d})$.

in [Guillamon et al., 2015] for the symmetric case, would not allow to continue the curve of periodic orbits.

To pick an initial condition for $Q$ and $x$ we use the condition for the existence of periodic orbits derived in (10) and (11), and then we integrate several orbits with random initial conditions until we detect the convergence to a periodic orbit.

Once we have obtained a periodic orbit, for an initial value of $Q$ (see Appendix Section 5.3) we proceed by continuation with respect of the parameter $Q$ (see Section 5.4 in the Appendix). In this computational part, we need to take care of the step size and of the number of continuation steps. It turns out that, after passing by the bifurcation point $Q_{P O}$, the continuation method follows the unstable periodic orbit. For every $A$, there exists a value of $Q$ for which this unstable periodic orbit passes so close to the hyperplanes $x_{i}=0$ that the Newton method we use does not converge. Thus, as $Q_{P O}$ depends on $A$ and $\delta$, the number of continuation steps needs to be adapted for every $(A, \delta)$ pair.

The curve of equilibrium points has been computed isolating $Q$ in the expression for $x$, that is, from $x_{ \pm}=\left((Q-2 \delta) \pm \sqrt{(Q-2 \delta)^{2}-20 A(1-Q)}\right) / 10$.

In the next section, we exploit the numerical implementation explained above to plot and compare the bifurcation curves.

\subsection{Impact of asymmetry on bifurcation gaps}

Here we extend the numerical study conducted in [Guillamon et al., 2015] for symmetric hypercycles to asymmetric ones. As mentioned, asymmetric hypercycles might be more realistic from a biological point of view since mutations giving place to different templates may produce differential fitness effects. We 


\begin{tabular}{|c|c|c|}
\hline \multicolumn{3}{|c|}{$Q_{S S}-Q_{P O}$} \\
\hline & $a a A A A$ & $a A a A A$ \\
\hline$\delta=0$ & $5.98054 \times 10^{-5}$ & $5.98054 \times 10^{-5}$ \\
$\delta=0.001$ & $6.12641 \times 10^{-5}$ & $6.01082 \times 10^{-5}$ \\
$\delta=0.01$ & $1.93241 \times 10^{-4}$ & $8.33985 \times 10^{-5}$ \\
$\delta=0.05$ & $2.34217 \times 10^{-3}$ & $4.96334 \times 10^{-4}$ \\
$\delta=0.1$ & $4.88186 \times 10^{-3}$ & $1.28505 \times 10^{-3}$ \\
\hline
\end{tabular}

Table 1: Bifurcation gaps for the cases $a a A A A$ and $a A a A A$ with different degrees of asymmetries, $\delta$, using $A=0.5$.

know explicitly $Q_{S S}$, the value of $Q$ for which the saddle-node (s-n) bifurcation of fixed points occurs, and we want to compute the value of $Q$ for which the s-n bifurcation of periodic orbits takes place, given by $Q_{P O}$. In [Guillamon et al., 2015] the authors observed that there is a slight difference between these two values, which they called gap. Here, we explore whether such a gap exists in the 5 -member asymmetric case and, if so, how it behaves as $a$ and $A$ differ from each other, i.e., as $\delta$ changes.

Figure 3 shows the two bifurcation curves (equilibria and periodic orbits) in terms of $Q$, taking $A=0.5$, as in [Guillamon et al., 2015], and increasing $\delta$ from 0.001 to 0.1 in the four panels. We observe in all cases a clear difference in the position of the bifurcation turning points, with $Q_{P O}>Q_{S S}$.

By definition of $\delta$, the larger it is, the more the hypercycle differs from the symmetric one. We are interested in quantifying how does the gap between the two bifurcation points behave when the system gets more asymmetric. For the symmetric case it was shown in Guillamon et al. [2015] that the gap reached a value of approximately $1.5 \times 10^{-4}$ for a certain value of parameter $A$ (see Figure 4, included here for the sake of comparison). In Figures 5 and 6 we can appreciate that the magnitude of the gap for the asymmetric cases increases with $\delta$ for both configurations, $a a A A A$ and $a A a A A$. Hence, as the hypercycle gets "more asymmetric", the difference between the values where the bifurcations of periodic orbits and fixed points occur is larger (see Table 1 where the value $Q_{P O}-Q_{S S}$ is displayed for each continuation curve). For example, when $\delta=$ 0.1 , the gap becomes about two orders of magnitude larger compared to the symmetric case.

Here, we make a necessary observation about errors, since we produce numbers that have been obtained numerically. In the computer codes we have written, the main sources of error are the numerical integrator and the Newton iterations. We have used a Runge-Kutta-Fehlberg method of orders 7-8 with local relative tolerance of $10^{-14}$. For the Newton method we have iterated until a tolerance of $0.5 \times 10^{-14}$ has been reached. 


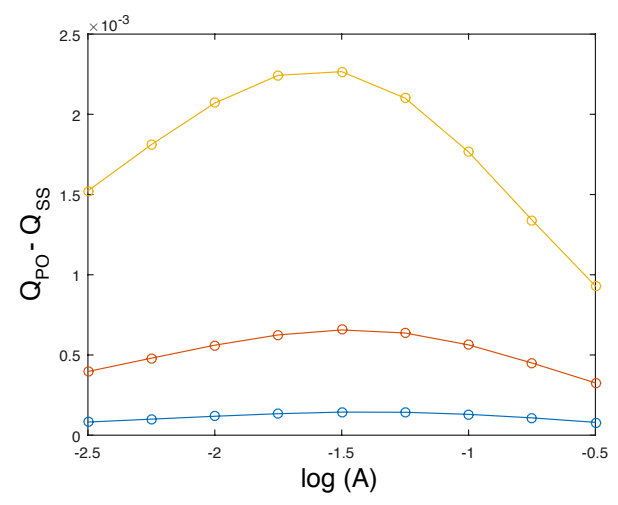

Figure 7: Distance between the saddle-node bifurcations of fixed points and of periodic orbits, $Q_{S S}-Q_{P O}$, for hypercycles with $n=5$ (blue), $n=6$ (red), and $n=8$ (orange) members considering symmetric Malthusian replication rates.

\subsection{Impact of higher dimensions on bifurcation gaps}

In this section we further analyse the bifurcation gap in terms of $Q$ for larger hypercycles focusing on symmetric systems with $n=6$ and $n=8$ members. These two cases are significant. The case $n=6$ is the simplest dimension higher than $n=5$ and with different parity. On the other hand, in the symmetric case, it is known that for $n=4 k, k \in \mathbb{N}$, two eigenvalues of the nontrivial equilibria are purely imaginary, so that $n=8$ is a specially interesting case. There is one difficulty that one must overcome somehow when increasing the dimension. That is, as numerical computations indicate, the coordinates of the periodic orbit pass extremely close to the origin. Then, computing the derivatives of the Poincaré map using:

$$
\frac{\partial P_{i}(x)}{\partial x_{j}} \approx \frac{P_{i}\left(x+h e_{j}\right)-P_{i}\left(x-h e_{j}\right)}{2 h},
$$

it might happen that $x-h e_{j}$ has some negative component, in which case we are not anymore in the simplex $\Omega_{1}$ and the flow will probably never come back to it (even if it did and crossed the Poincaré section again the solution would have nothing to do with the expected one). One could think that decreasing $h$ would solve the problem, but doing so will lead to a huge numerical error in the derivative (as it happens when we have a small value in the denominator). This is why we have been forced to use more accurate algorithms to obtain the derivative of the Poincaré map in order to apply successfully the Euler-Newton continuation method. This alternative way is explained in Appendix Section 5.4 .

The actual computations look for the value of $Q$ along the continuation process at which the sign of the derivative of $Q$ with respect to the arc length of the curve which gives the periodic orbit with respect to the parameter $Q$ changes 
and then compare it with the value of $Q$ corresponding to the s-n bifurcation for the fixed points which is $Q=2(\sqrt{n A(1+n A)}-n A)$. As long as we use a small step size when applying the Euler-Newton method, the continuation goes on (for example using as step size $10^{-6}$, which is what we have done).

The analyses for $n=6$ and $n=8$ reveal that the results are not only analogous to the case $n=5$ (see Fig. 4) but also that the width of the gap increases with the dimension of the hypercycle (see Fig. 7). More specifically, this distance increases more than one order of magnitude from $n=5$ to $n=8$. Notice that when $n=6$ (red curve, Fig. 7), the increase of the Malthusian replication rate makes the gap to slightly grow up to $\log (A)=-1.5$. Then the gap slightly decreases. Within all the range of $A$ analyzed, the size of the gap remains at $Q_{S S}-Q_{P O} \approx 0.5 \times 10^{-3}$, while this distance for the symmetric $n=5$ case was about $1.5 \times 10^{-4}$ in its largest value tuning $A$ (see Fig. 4 and [Guillamon et al., 2015]). A similar behavior is found for $n=8$, being the largest value of the bifurcation gap $Q_{S S}-Q_{P O} \approx 2.25 \times 10^{-3}$.

\section{Conclusions}

In this manuscript we have conducted an analytical study of a general $n$-member, asymmetric hypercycle with the Malthusian replication constants: $a A \ldots A$. We have found the coexistence fixed points, their bifurcation values and the region in which the vector field can have periodic orbits. We have then focused on cases $a a A A A$ and $a A a A A$ for five-member hypercycles. In this way we have exhausted all of the possible asymmetries, relabelling constants and/or variables if necessary.

Following previous results on oscillating 5-member hypercycles [Guillamon et al., 2015], we have checked that the gap found between the saddle-node (s-n) bifurcation value of fixed points and the s-n bifurcation value of periodic orbits in symmetric hypercycles also holds in asymmetric ones. Actually, the gap grows as the asymmetry of the system gets larger. These results strengthen the "gap problem" introduced in [Guillamon et al., 2015], as it was identified in the symmetric case, which is a less realistic case from a biological point of view. Moreover, in the symmetric case, the gap is shown to increase for larger hypercycles. Symmetric hypercycles assume that all of the members of the hypercycle are neutral mutants, since they might be synthesized from previously existing templates by replication and mutation processes. In this sense, recent experimental results on mutational fitness effects for RNA viruses quantified neutral spontaneous mutations happening with about $25 \%$ of probability [Sanjuán et al., 2004; Carrasco et al., 2007]. Hence, even for small hypercycles, asymmetries might be expected.

The biological implications of these gaps could be relevant within the framework of so-called delayed transitions [Sardanyés \& Solé, 2005; Sardanyés \& Solé, 2006]. It is known that after a s-n bifurcation a saddle remnant (also named ghost) appears in the phase space [Strogatz, 2000; Fontich \& Sardanyés, 2008; ,Duarte et al., 2012; Strogatz \& Westervelt, 1989; Sardanyés \& Solé, 2005]. In 
this phenomenon, the flow takes a long-lasting excursion just after the s-n bifurcation before going to the only asymptotically globally stable attractor, which involves extinction. This phenomenon was interpreted as a kind of memory for hypercycles occurring after the bifurcation [Sardanyés \& Solé, 2005], possibly becoming a selective advantage in fluctuating environments [Sardanyés \& Solé, 2005]. Interestingly, this delaying phenomenon has been described in models for delayed switching of charge-density waves [Strogatz \& Westervelt, 1989], as well as in an experimental electronic circuit behaving as a Duffing oscillator [Trickey \& Virgin, 1998]. Since the gap described in this article is found in between two different s-n bifurcations, two delayed transitions may be found, one when $Q \lesssim Q_{P O}$ and another when $Q \lesssim Q_{S S}$. The presence of two consecutive s-n bifurcations at decreasing $Q$ could involve an enhancement of the delaying effects and thus a higher memory capacity of the system, therefore further slowing down hypercycles' extinction. However, we must notice that delayed transition phenomena are local. This means that the delaying effect of the saddle remnant occurs with parameter values close to the bifurcation value. Despite this fact, future research could be devoted to quantify the delaying times within the region $\left[Q_{S S}, Q_{P O}\right]$ in order to analyse whether a reinforcement of the delayed transitions occurs, especially under the presence of catalytic parasites.

\section{Acknowledgements}

The research leading to these results has received funding from "la Caixa" Foundation. This work has been partially funded by the Spanish grants MINECO MTM2013-41168-P, MTM2016-80117-P (MINECO/FEDER, UE) (EF) and MTM201571509-C2-2-R (AG), and the Catalan grants AGAUR 2014SGR-1145 (EF) and 2014SGR-504 (AG). JS has been partially funded by the CERCA Programme of the Generalitat de Catalunya.

\section{References}

[1] Eigen, M. \& Schuster, P. [1979] The Hypercycle. A Principle of Selforganization. Springer-Verlag, Germany.

[2] Allgower, E.L. \& Kurt, G. [1987] Introduction to Numerical Continuation Methods, Society for Industrial and Applied Mathematics.

[3] Eigen, M., [1971] "Selforganization of Matter and the Evolution of Biological Macromolecules," Die Naturwissenschaften, 58, 465-523.

[4] Campos, P.R.A. Fontanari, J.F. \& Stadler, P.F. [2000] "Error propagation in the hypercycle," Phys. Rev. E, 61, 2996-3002.

[5] Smith, J.M. \& Szathmáry, E. [1995] The major transitions in evolution. Oxford University press, Great Britain. 
[6] Sardanyés, J. [2009] Landscape ecology research trends, [Chapter 6. The Hypercycle: from molecular to ecosystems dynamics]. Nova Publishers.

[7] Sardanyés, J. \& Solé, R.V. [2005] "Ghosts in the origins of life?," Int. J. of Bifurc. and Chaos, 16(9), 2761-2765.

[8] Nuño, J.C. Montero, F. \& de la Rubias, F. J. [1993] "Influence of external fluctuations on a hypercycle formed by two kinetically indistinguishable species," J. theor. Biol., 165, 553-75.

[9] Silvestre, D.A.M.M. \& Fontanari, J. F. [2008] "The information capacity of hypercycles," J. Theor. Biol., 254, 804-806.

[10] Solé, R. V. Saldanya, J. Montoya, J. M. \& Erwin, D. H. [2010] "Simple model of recovery dynamics after mass extinction," J. Theor. Biol., 267(2), $193-200$.

[11] Groenenboom, M.A.C. \& Hogeweg, P. [2002] "Space and the persistence of male-killing endosymbionts in insect populations," Proc. R. Soc. Lond. B, 269, 2509-2518.

[12] Guillamon, A. Fontich, E. \& Sardanyés, J. [2015] "Bifurcations analysis of oscillating hypercycles". J. Theor. Biol., 387, 23-30.

[13] Hofbauer, J. Mallet-Paret, J. \& Smith, H.L. [1990] "Stable Periodic Solutions for the Hypercycle System." J. Dyn. and Diff. Equations, 3(3), $423-436$.

[14] Farkas, M. [2001] Dynamical Models in Biology. Academic Press, San Diego.

[15] Kauffman, S.A. [1993] The origins of order, [Chapter 9. Hypercycles and coding]. Oxford University Press.

[16] Sardanyés, J. \& Solé, R.V., [2006] "Bifurcations and phase transitions in spatially-extended two-member hypercycles." J. Theor. Biol., 234(4), 468482.

[17] Sardanyés, J. \& Solé, R.V. [2007] "Spatio-temporal dynamics in simple asymmetric hypercycles under weak parasitic coupling." Physica D, 231(2), 116-129.

[18] Sardanyés, J. \& Solé, R.V. [2007] "Delayed transitions in nonlinear replicator networks: About ghosts and hypercycles." Chaos, Solitons and Fractals, 31(2): 313-319.

[19] Simó, C. [1990] On the analytical and numerical approximation of invariant manifolds, in Les méthodes modernes de la mécanique., 285-329, Editions Frontieres, Paris. 
[20] Sardanyés, J. [2009] Dynamics, Evolution and Information in Nonlinear Dynamical Systems of Replicators. Doctoral Thesis. Universitat Pompeu Fabra, Spain.

[21] Sanjuán, R., Moya, A. \& Elena, S.F. [2004] "The distribution of fitness effects caused by single-nucleotide substitutions in an RNA virus". Proc. Natl. Acad. Sci. U.S.A., 101, 8396-8401.

[22] Carrasco, P. de la Iglesia, F. \& Elena, S. F. [2007] "Distribution of fitness and virulence effects caused by single-nucleotide substitutions in Tobacco etch virus." J. Virol., 81, 12979-12984.

[23] Strogatz, S.H. [2000] Nonlinear dynamics and chaos. Westview Press.

[24] Fontich \& Sardanyés [2008] "General scaling law in the saddle-node bifurcation: a complex phase space study." J. Phys. A: Math. Theor., 41, 015102.

[25] Duarte, J. Januário, Martins, N., \& Sardanyés, J. [2012] "Scaling law in saddle-node bifurcations for one-dimensional maps: a complex variable approach". Nonlinear Dyn., 67: 541-547.

[26] Strogatz, S.H. \& Westervelt, R.M. [1989] "Predicted power laws for delayed switching of charge-density waves", Phys. Rev. B, 40, 10501-10508.

[27] Trickey, S.T. \& Virgin, L. N. [1998] "Bottlenecking phenomenon near a saddle-node remnant in a Duffing oscillator". Phys. Lett. A, 248, 185-190.

\section{Appendices}

\subsection{Poincaré maps}

Poincaré maps are a powerful device to study both theoretical and computational aspects of vector fields. In this work we use them to compute periodic orbits of a vector field $F$ which correspond to fixed points or periodic points of $P$, the Poincaré map associated to $F$ and some section. In its turn fixed points of $P$ correspond to zeros of the map $H$, defined as

$$
H(x):=P(x)-x .
$$

Thus in this way we reduce the problem of obtaining periodic orbits of vector fields to the problem of finding zeros of functions. To define a Poincaré map first we have to choose a Poincaré section, $\Sigma_{P}$. Motivated by the fact that, after the bifurcation, our systems have two interior equilibrium points we just choose a section defined by the first coordinate fixed constant at a value which is the midpoint between the two first coordinates of these equilibrium points (whenever they exist). Hence we take

$$
\Sigma_{P}:=\left\{x_{1}=\frac{Q}{2 n}\right\}
$$


in the symmetric case and

$$
\Sigma_{P}:=\left\{x_{1}=\frac{Q-2 \delta}{10}+\delta\right\},
$$

in the $a a A A A$ case and $n=5$, and

$$
\Sigma_{P}:=\left\{x_{1}=\frac{Q-2 \delta}{10}\right\},
$$

in the $a A a A A$ case and $n=5$.

We note that we could have chosen any other $x_{i}$ coordinate and proceed analogously. Then taking an initial condition in $\Sigma_{P}$ we integrate our vector field $F$ until we reach again $\Sigma_{P}$ crossing it in the same sense as the vector field crosses $\Sigma_{P}$ at the initial point $x$. To detect the case for which the orbit of $x \in \Sigma_{P}$ does not return to $\Sigma_{P}$ we prescribe a time limit of integration. For the numerical integration we use a method based on two Runge-Kutta-Fehlberg algorithms of orders 7 and 8 (RKF78) with automatic step size control, using a step size $10^{-4} \leq \Delta t \leq 10^{-1}$ and local relative tolerance $10^{-14}$. When we detect that the orbit has crossed $\Sigma_{P}$ in the desired sense, we proceed to obtain the point of the trajectory that is on $\Sigma_{P}$. For that we use the Newton method to find a zero of the function

$$
\Phi(t)=y_{1}(t)-\frac{Q}{2 n},
$$

where $y_{1}$ denotes the first coordinate of the orbit (which we compute using the RKF78 method). The Newton scheme gives the sequence of time iterates

$$
t_{k+1}=t_{k}-\frac{y_{1}\left(t_{k}\right)-\frac{Q}{2 n}}{\dot{y}_{1}\left(t_{k}\right)} .
$$

From this, we get the next step size $h_{k+1}=t_{k+1}-t_{k}$ to perform one more step of the RKF78 integration and arrive closer to $\Sigma_{P}$. We repeat this iteration until a prefixed tolerance (set as $\varepsilon=0.5 \times 10^{-14}$ ) is reached. As a result of this process, we get a point that is on the orbit and on $\Sigma_{P}$ as well, which taken as $P(x)$, the image of $x$ by the Poincaré map.

\subsection{Derivative of the Poincaré map}

In this section we provide a formula for the differential of the Poincaré maps we use in this work, which is based upon the solution of the variational equation associated to the vector field. It provides a much more accurate way to effectively compute the differential than to approximately compute the partial derivatives through quotiens of differences. Let $F: \Omega \subset \mathbb{R}^{n} \longrightarrow \mathbb{R}^{n}$ be a vector field and $\varphi(t, x)$ its flow. The variational equation is

$$
\frac{d}{d t} \Phi(t, x)=D F(\varphi(t, x)) \Phi(t, x) .
$$


Let $M(t, x)$ be the solution of (15) with initial condition $M(0, x)=$ Id. It is well known that $M(t, x)=D_{x} \varphi(t, x)$. Given a section $\Sigma_{P}=\left\{x_{1}=c\right\}$, the associated Poincaré map $P$ is

$$
P(y)=\hat{\varphi}(\tau(\hat{x}), \hat{x}), \quad \hat{x}=\left(c, y_{2}, \ldots, y_{n}\right) \in \Sigma_{P},
$$

with $y=\left(y_{2}, \ldots, y_{n}\right), \hat{\varphi}=\left(\varphi_{2}, \ldots, \varphi_{n}\right)$, and $\tau(\hat{x})$ being the time needed for the solution to arrive to $\Sigma_{P}$ as described in Section 5.1. Now we can compute the differential of $P$ by using the chain rule

$$
D P(y)=\frac{\partial \hat{\varphi}}{\partial t}(\tau(\hat{x}), \hat{x}) D_{y} \tau(\hat{x})+D_{x} \hat{\varphi}(\tau(\hat{x}), \hat{x}) D_{y} \hat{x} .
$$

Let $f_{j}=F_{j}(\varphi(\tau(\hat{x}), \hat{x}))$ and $m_{i, j}=M_{i, j}(\tau(\hat{x}), \hat{x})$. Using this notation we have

$$
\begin{gathered}
\frac{\partial \hat{\varphi}}{\partial t}(\tau(\hat{x}), \hat{x})=\left(f_{2}, \ldots, f_{n}\right)^{\top}, \\
\mathrm{D}_{x} \hat{\varphi}(\tau(\hat{x}), \hat{x})=\left(\begin{array}{ccc}
m_{2,1} & \ldots & m_{2, n} \\
\vdots & & \vdots \\
m_{n, 1} & \ldots & m_{n, n}
\end{array}\right),
\end{gathered}
$$

and

$$
\mathrm{D}_{y} \hat{x}=\left(\begin{array}{c}
0 \\
\operatorname{Id}_{\mathrm{n}-1, \mathrm{n}-1}
\end{array}\right) .
$$

Since $\tau$ is characterized by the implicit condition

$$
\varphi_{1}(\tau(\hat{x}), \hat{x})=c,
$$

we can obtain its derivative by differentiating both sides of Eq. (16). We have

$$
\frac{\partial \varphi_{1}}{\partial t}(\tau(\hat{x}), \hat{x}) D_{y} \tau(\hat{x})+D_{x} \varphi_{1}(\tau(\hat{x}), \hat{x}) D_{y} \hat{x}=0
$$

so that

$$
\begin{aligned}
\mathrm{D}_{y} \tau(\hat{x}) & =-\frac{1}{f_{1}}\left(m_{1,1}, \ldots, m_{1, n}\right)\left(\begin{array}{c}
0 \\
\operatorname{Id}_{\mathrm{n}-1, \mathrm{n}-1}
\end{array}\right) \\
& =-\frac{1}{f_{1}}\left(m_{1,2}, \ldots, m_{1, n}\right),
\end{aligned}
$$

and finally

$$
\operatorname{DP}(y)=\left(\begin{array}{ccc}
m_{2,2}-\frac{f_{2}}{f_{1}} m_{1,2} & \ldots & m_{2, n}-\frac{f_{2}}{f_{1}} m_{1, n} \\
\vdots & & \vdots \\
m_{n, 2}-\frac{f_{n}}{f_{1}} m_{1,2} & \ldots & m_{n, n}-\frac{f_{n}}{f_{1}} m_{1, n}
\end{array}\right) .
$$

Therefore to apply this formula we have to integrate both the equation and the variational equation from the initial condition $\hat{x}=\left(c, x_{2}, \ldots, x_{n}\right) \in \Sigma_{P}$ until we arrive again at $\Sigma_{P}$ crossing it in the same sense to obtain $\varphi(\tau(\hat{x}), \hat{x}), f_{j}$, and $m_{i, j}$. 


\subsection{Search of a periodic orbit}

As explained in Section 5.1. we have to find the zeros of $H(x)=P(x)-x$. For that we use the Newton algorithm, which, given an initial guess $x^{0}$, is defined by the following iteration:

$$
x^{k+1}=x^{k}-D H\left(x^{k}\right)^{-1} H\left(x^{k}\right) .
$$

We have $D H(x)=D P(x)-$ Id. We can compute $D P(x)$ as explained in the previous section or using the central differences method, using, for example, $\delta_{x}=10^{-4}$. To solve (17) we rewrite it as the linear system

$$
D H\left(x^{k}\right)\left(x^{k+1}-x^{k}\right)=-H\left(x^{k}\right),
$$

and we perform a LU decomposition of the matrix $D H\left(x^{k}\right)$ to easily solve it. We iterate (17) until we get $k$ such that $\left\|x^{k+1}-x^{k}\right\|<\varepsilon$ (with $\varepsilon=0.5 \times 10^{-14}$ ) or until the maximum number of iterates is exceeded, in which case we decide that the method does not converge. In the first case, we consider $x^{k+1}$ as a fixed point of the Poincaré map, $P$, and so, a point belonging to a periodic orbit of the vector field $F$.

\subsection{Continuation of periodic orbits: The Euler-Newton method}

The Euler-Newton method is a method of continuation of implicit curves given by the zeros of some function $f: \mathbb{R}^{n+1} \rightarrow \mathbb{R}^{n}$ of class $C^{r}$ with $r \geq 1$ once we know a point $x^{*} \in \mathbb{R}^{n+1}$ such that $f\left(x^{*}\right)=0$. The whole idea of this method consists in finding the derivative of the implicit curve (we know that it exists locally if rank $\left(\mathrm{D} f\left(x^{*}\right)\right)=n$ by the Implicit Function Theorem), apply the Euler method to find a first approximation of the following point of the curve in the desired direction and then refine this approximation using a Newton-like method. A more detailed explanation of how does this method work can be found in [Simó, 1990].

If we denote $g: I \subseteq \mathbb{R} \rightarrow \mathbb{R}^{n+1}$ the implicit curve parameterized by the arc length, then it can be proved that

$$
\frac{d g_{j}}{d s}=(-1)^{j} \frac{A_{j}}{\sqrt{\sum_{k=0}^{k=n} A_{k}^{2}}},
$$

where $A_{j}$ is the determinant of $D f\left(x^{*}\right)$ without the $j$-th column. It can also be proved that then the sequence $\left\{x_{i}\right\}_{i}$ defined by

$$
x_{i+1}=x_{i}-\mathrm{D} f\left(x_{i}\right)^{\top}\left(\mathrm{D} f\left(x_{i}\right) \mathrm{D} f\left(x_{i}\right)^{\top}\right)^{-1} f\left(x_{i}\right),
$$

where $x_{0}=x^{*}+h \nabla g$, does converge to $\left\{x \in \mathbb{R}^{n+1} \mid \exists t \in I\right.$ with $\left.g(t)=x\right\}$ if $h$ is small enough (see [Allgower \& Kurt , 1987]). To obtain the whole curve as long as it is regular we only have to apply these steps repeatedly. 
As we have already mentioned in earlier sections, our particular interest in this method is to apply it to do the continuation of fixed points for the Poincaré map with respect to parameters and therefore to study bifurcations of periodic orbits. We apply the method to $f(x, Q)=P_{Q}(x)-x$, where $P_{Q}$ is the Poincaré map depending on the parameter $Q$. 